This is the submitted version of the article:

Singhal D., Paterson J., Ben-Khedim M., Tainoff D., Cagnon L., Richard J., Chavez-Angel E., Fernandez J.J., Sotomayor-Torres C.M., Lacroix D., Bourgault D., Buttard D., Bourgeois O.. Nanowire forest of pnictogen-chalcogenide alloys for thermoelectricity. Nanoscale, (2019). 11. : 13423 - . $10.1039 /$ c9nr01566c.

Available at: https://dx.doi.org/10.1039/c9nr01566c 


\title{
Nanowire Forest of Pnictogen-Chalcogenide alloys for Thermoelectricity
}

\author{
Dhruv Singhal, ${ }^{\dagger, \ddagger}, \mathbb{\uparrow}$ Jessy Paterson, ${ }^{\ddagger}, \boldsymbol{\Phi}$ Meriam Ben-Khedim, ${ }^{\ddagger}, \boldsymbol{\Phi}, \S$ Dimitri \\ Tainoff,,$\uparrow$ Laurent Cagnon,

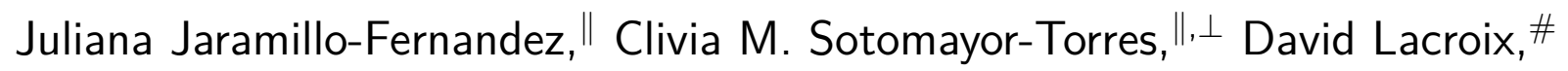 \\ Daniel Bourgault,,+9 Denis Buttard, ${ }^{\dagger} \uparrow$ and Olivier Bourgeois $*,+, \uparrow$ \\ $\dagger$ †niv. Grenoble Alpes, CEA, INAC-Pheliqs, F-38000 Grenoble, France \\ $\ddagger$ Institut NÉEL, CNRS, 25 avenue des Martyrs, F-38042 Grenoble, France \\ IUniv. Grenoble Alpes, Grenoble, France \\ $\S$ Technology R\&SD, STMicroelectronics, 13106 Rousset, France \\ \|Catalan Institute of Nanoscience and Nanotechnology (ICN2), CSIC and BIST, Campus \\ UAB, Bellaterra 08193 Barcelona, Spain \\ $\perp$ ICREA, Passeig Lluís Companys 23, 08100 Barcelona, Spain \\ \#Université de Lorraine LEMTA - Laboratoire dÉnergie et de Mécanique Théorique et \\ Appliquée - CNRS UMR 7563 ENSEM - 2 avenue de la Forêt de Haye BP 9016154505 \\ Vanduvre les Nancy, cedex - FRANCE \\ E-mail: olivier.bourgeois@neel.cnrs.fr
}

\begin{abstract}
Pnictogens and chalcogenides compounds have been seen as high-potential materials for efficient thermoelectric conversion over the past few decades. It is also believed that with nanostructuration, the properties of these pnictogen-chalcogenides
\end{abstract}


compounds can be further enhanced towards a more efficient heat conversion. Here, we report reduced thermal conductivity of a large ensemble of $\mathrm{Bi}_{2} \mathrm{Te}_{3}$ alloy nanowires with selenium for n-type and antimony for p-type $\left(\mathrm{Bi}_{2} \mathrm{Te}_{3-y} \mathrm{Se}_{y}\right.$ and $\mathrm{Bi}_{2-x} \mathrm{Sb}_{x} \mathrm{Te}_{3}$ respectively). The nanowire growth was carried out through electrodeposition in nanoporous aluminium oxide templates with high aspect ratios- leading to high density $\left(10^{9} / \mathrm{cm}^{2}\right)$ of nearly-identical nanowires. The temperature dependence of thermal conductivity for the nanowire ensembles was acquired through a highly-sensitive $3 \omega$ measurement technique. The change in the thermal conductivity of nanowires is largely affected by the surface roughness rather than the presumed confinement effect due to enhanced boundary scattering. The major factor that influences the thermal conductivity was found to be the ratio of the rms roughness to the correlation length of the nanowire. With Seebeck coefficient and electrical conductivity at room temperature, the overall thermoelectric figure of merit, $Z T$, of the nanowire ensemble was calculated with a maximum value of 0.3 at $300 \mathrm{~K}$ for the $\mathrm{Bi}_{1.55} \mathrm{Te}_{2.9} \mathrm{Se}_{0.55}$ sample.

Despite being considered undesirable in most of the cases, waste heat happens to be one of the most significant source for energy recovery. Significant growth of interest has been seen over the last few decades for developing the field of recapturing waste heat for generating electricity from a gradient of temperature using the Seebeck effect. ${ }^{1-3}$ For this purpose, a new generation of thermoelectric generators (TEGs) is emerging, capable of this energy conversion, that are made up of nanomaterials that allow the generation of electricity even at the nanoscale. ${ }^{4-7}$

The figure of merit of a thermoelectric (TE) material is characterized by a dimensionless parameter, $Z T=\frac{\sigma S^{2} T}{\kappa}$, where $\sigma$ is the electrical conductivity, $S$ is the Seebeck coefficient, $T$ is the temperature and $\kappa$ is the thermal conductivity. The reduction in thermal conductivity through phonon engineering alongside high power factor $\left(\sigma S^{2}\right)$ in the materials are being employed for enhanced thermal cooling and power generation with increased efficiency for energy harvesting. ${ }^{8}$ Extensive research is undergoing to find new thermoelectric materials for large-scale practical applications by two means: (1) structural engineering of existing 
materials for reduced thermal transport or (2) development of new complex materials with inherently enhanced TE properties. ${ }^{9,10}$

The best candidates for high $Z T$ materials are often nanostructured doped semiconductors. Among them, typically used TE materials are pnictogen-chalcogenides alloys as they exhibit the highest $Z T$ for bulks at room temperature; ${ }^{9,11}$ the most common commercial application being provided by bismuth telluride and its alloys (with selenium and antimony) over the temperature ranges of 200-400 K. ${ }^{9}$ The layered crystal structure of $\mathrm{Bi}_{2} \mathrm{Te}_{3}$ acts as natural scattering points for phonons inducing a low thermal conductivity. At the same time, it also produces a narrow electronic gap with six degenerated valleys which leads to good electrical conductivity, thus making the family of $\mathrm{Bi}_{2} \mathrm{Te}_{3}$ compounds attractive for thermoelectric materials. If experimental evidence of reduced thermal conductivity has been shown with $40 \mathrm{~nm}$ diameter nanowire of $\mathrm{Bi}_{2} \mathrm{Te}_{3}$ compared to the bulk value, ${ }^{12-15}$ no experimental work has been dedicated to the temperature variation of the thermal conductivity of nanowires the different $\mathrm{n}$ and $\mathrm{p}$ alloys. Indeed, there exists the possibility to tune the phononic contribution of the thermal transport by reducing the dimensions of the system. In this context, nanowires have been one of the most promising contender for obtaining low thermally conductive materials with state-of-the-art high electronic conductivity and Seebeck coefficients, a step towards electron-crystal phonon glass systems. ${ }^{16,17}$ In the past, numerous studies have been carried out for various types of individual nanowires, however, these measurements depend significantly on the physical and chemical properties of the particular nanowire under study. Consequently, there is high probability of finding variations between nano-objects as it arises from the study of sole systems. Hence, it is evidently detrimental to measure single object for the demonstration of efficiency of macroscopic scale TE device involving a significant large number of nanowires.

Therefore, in this work, the major objective is to investigate the thermal properties of a very large assembly of $\mathrm{Bi}_{2} \mathrm{Te}_{3-y} \mathrm{Se}_{y} / \mathrm{Bi}_{2-x} \mathrm{Sb}_{x} \mathrm{Te}_{3}$ alloy nanowires embedded in nanoporous alumina template (AAO). Alumina holey membrane are thought to be one of the ideal 
building block of a new generation of TE module because of its capability to serve as a template for the growth of highly ordered embedded nanostructures. The AAO templates provides a mechanical support to nanowires with very high-aspect ratios. Indeed, dense forests of nanowires can be a potential architecture for TE devices where each nanowires act as a leg of the thermoelectric generator, given that appropriate connections between differently doped nanowires are made. ${ }^{5,18-20}$ The dense forest of $\mathrm{Bi}_{2} \mathrm{Te}_{3-y} \mathrm{Se}_{y} / \mathrm{Bi}_{2-x} \mathrm{Sb}_{x} \mathrm{Te}_{3}$ alloy nanowires with constant diameter is grown-through the bottom-up growth technique- by electrodeposition inside the nanoporous anodic aluminium oxide templates, where the geometry of nanowires is determined by the internal pore geometry. ${ }^{13,21-23}$ The thermal properties of these forests of nanowires are measured in the same configuration as the proposed architecture for TE modules or energy harvesting systems. The thermal conductivity is determined by using the $3 \omega$ method adapted to anisotropic media. ${ }^{24}$ The observed reduction of thermal conductivity as compared to the bulk is related to the geometrical parameters of the nanowires viz. reduced diameter, grain size and surface roughness.

\section{Experimental Section}

The growth of embedded nanowire forest of pnictogen-chalcogenide alloys $\left(\mathrm{Bi}_{2} \mathrm{Te}_{3-\mathrm{y}} \mathrm{Se}_{\mathrm{y}}\right.$ for n-type and $\mathrm{Bi}_{2-\mathrm{x}} \mathrm{Sb}_{\mathrm{x}} \mathrm{Te}_{3}$ for p-type) was performed with the help of nanoporous anodic aluminium oxide templates, well-known for the fabrication of ordered nanostructures. Anodising of aluminium in acidic electrolytes leads to the formation of AAO templates. ${ }^{25}$ Based on the self-organising regime, they have been used for the synthesis of ordered nanostructures (Figure 1a shows top-view of AAO template used for the growth of nanowires) with high aspect ratios and tunable morphologies. Especially because of the relationship between its characteristic morphologies and the anodizing parameters (applied voltage, type, concentration and temperature of the electrolyte etc... ${ }^{26,27}$ Highly-dense arrays of $\mathrm{Bi}_{2} \mathrm{Te}_{3-\mathrm{y}} \mathrm{Se}_{\mathrm{y}}$ and $\mathrm{Bi}_{2-\mathrm{x}} \mathrm{Sb}_{\mathrm{x}} \mathrm{Te}_{3}$ nanowires were grown by the method of electro-deposition in these AAO templates (descrip- 


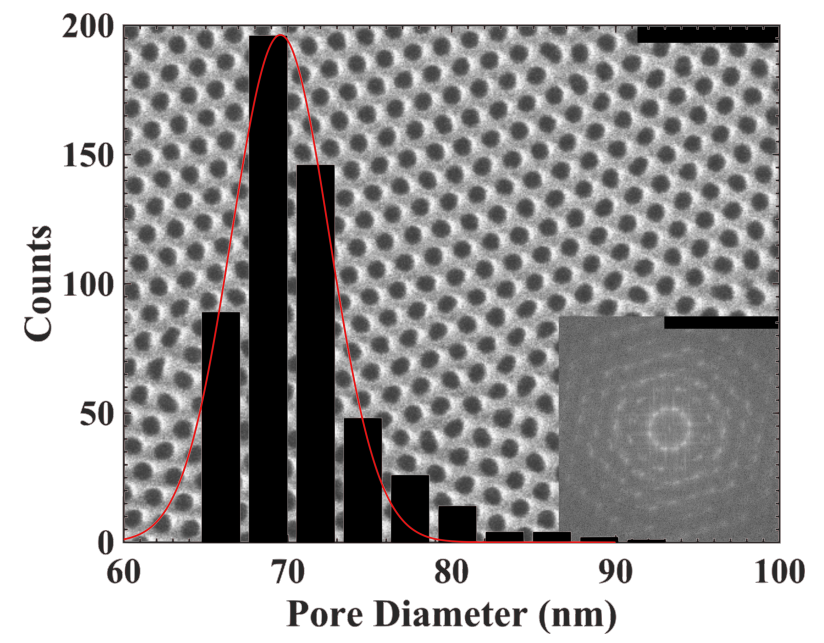

(a)
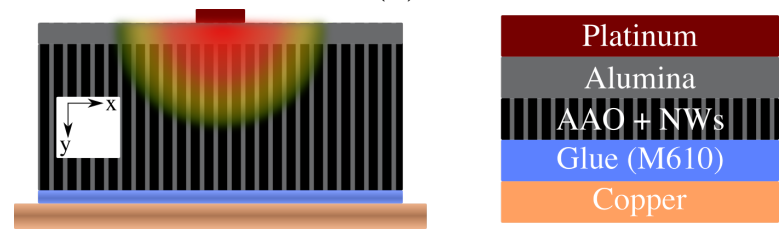

(b)

Figure 1: (a) Pore diameter histogram with the inset of SEM micrograph (scale is $500 \mathrm{~nm}$ ) of the top-view of AAO with FFT for calculating the mean interpore distance $(100 \mathrm{~nm})$. The maximum is located around $70 \mathrm{~nm}$.(b) Schematic of different layers in the sample used for studying the thermal properties of the nanowires.

tion of the fabrication procedure is detailed in Supporting information). Figure $1 b$ shows the structure of embedded nanowires with different layers deposited for carrying out thermal measurements.

The high resolution transmission electron microscopy (HRTEM) revealed that the asgrown nanowires polycrystalline in nature textured along the growth directions of [0 1 5], [l $\left.\begin{array}{lll}1 & 0 & 10\end{array}\right]$ and [ $\left[\begin{array}{lll}1 & 1 & 0\end{array}\right]$. As their dimensions are homogeneous to a certain degree, the determined values are an averaged value over a large ensemble nanowires (around $0.06 \mathrm{~mm}^{2}$ ). Measuring embedded nanowires in the templates has practical advantages, primarily the ease in post-fabrication process compared to the challenging lithography steps involved in the single nanowire measurements. It also prevents the nanowires from coming in contact with air which leads to surface oxidation. Such surface oxidation might modify the transport properties of the nanowires, which can induce errors during single nanowire measurements. ${ }^{28}$ It is also 

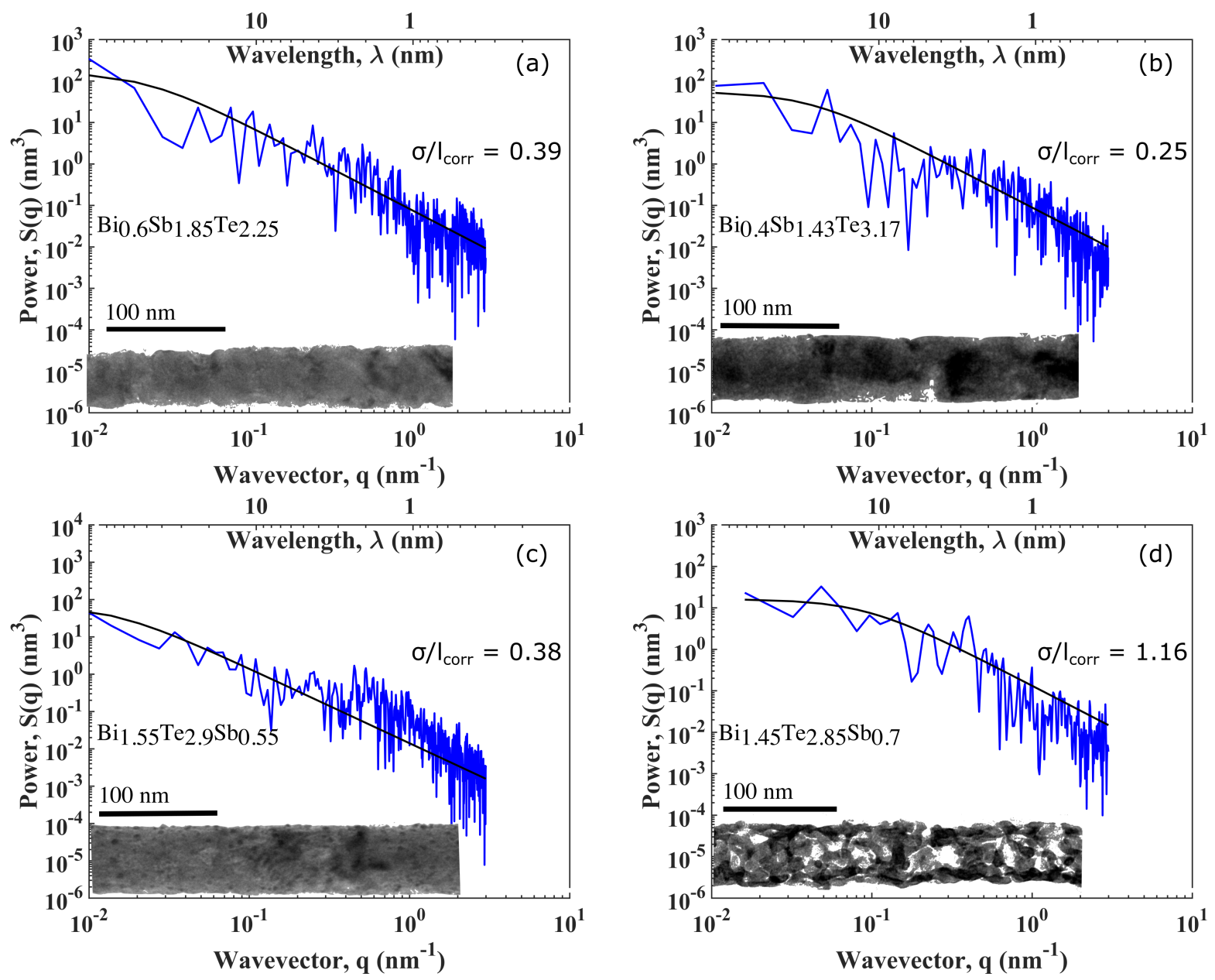

Figure 2: Extraction of the rms $(\sigma)$ roughness and correlation length $\left(l_{\text {corr }}\right)$ calculated from the HRTEM micrographs of single nanowires for (a) $\mathrm{Bi}_{0.6} \mathrm{Sb}_{1.85} \mathrm{Te}_{2.55}$ nanowires electrodeposited at $-0.3 \mathrm{~V}\left(\sigma=3.5 \mathrm{~nm}, l_{\text {corr }}=8.8 \mathrm{~nm}\right)$, (b) $\mathrm{Bi}_{0.4} \mathrm{Sb}_{1.43} \mathrm{Te}_{3.17}$ nanowires deposited at $-0.15 \mathrm{~V}\left(\sigma=2.2 \mathrm{~nm}, l_{\text {corr }}=5.7 \mathrm{~nm}\right)$, (c) $\mathrm{Bi}_{1.55} \mathrm{Te}_{2.9} \mathrm{Se}_{0.55}$ nanowires deposited at $0 \mathrm{~V}(\sigma=$ $\left.2.1 \mathrm{~nm}, l_{\text {corr }}=10.1 \mathrm{~nm}\right)$ and, (d) $\mathrm{Bi}_{1.45} \mathrm{Te}_{2.85} \mathrm{Se}_{0.7}$ nanowire deposited at $-0.2 \mathrm{~V}$ ( $\sigma=2.1 \mathrm{~nm}$, $\left.l_{\text {corr }}=1.8 \mathrm{~nm}\right)$.

worth mentioning that the embedded nanowires are encapsulated in AAO and thus the effect of radiation losses are insignificant, which is not the case in single nanowire measurements.

The crystal structure and the roughness of the nanowires are two crucial parameters for understanding the heat transport, both of which were extracted from the HRTEM images. The roughness is defined as the variation of the diameter around the mean value, at the atomic scale, along the main axis (y axis) A series of high-resolution images of the nanowires were acquired to ascertain the average roughness on the nanowire surface and electron diffraction 
patterns at different points on the nanowire to understand the crystal structure along the growth axis of nanowires. The diameter of nanowires was also confirmed through the HRTEM images and was found to be within the error coming from the pore diameter in AAO. To extract the surface roughness of the nanowires from the high resolution images, firstly, a background subtraction was applied and then the power spectrum $S(q)$ was calculated from the averaged surface roughness amplitudes (over multiple nanowires).

In order to understand the scattering of phonons on a real surface, not only the roughness plays a role but also their correlation along the nanowire. This is done by calculating the power spectrum determination of the correlation length, a crucial number to understand phonon transport in $1 D$ nanosystems. ${ }^{29}$ The autocovariance function, ${ }^{30,31} C(y)$, of a random rough surface follows an exponential relation with the rms roughness $\sigma$ and the correlation length $l_{\text {corr }}$ with the following equation

$$
C(y)=\sigma^{2} \exp \left(\frac{-y}{l_{\text {corr }}}\right)
$$

The correlation length is a statistical parameter determining the decay of the autocovariance and related to the lateral length scale of the roughness. The Fourier transform of the autocovariance function renders to a Lorentzian form, which is actually the power spectrum by convolution theorem

$$
S(q)=2 l_{\text {corr }} \sigma^{2} \frac{1}{\left[1+\left(2 \pi l_{\text {corr }} q\right)^{2}\right]}
$$

The averaged power spectrum was then calculated by analysing various nanowires of the same (Figure 2). The relation between the power spectrum and the wavevectors $q$ was used to determine the correlation length by using Eq. 2. The mis rom and important rote in understanding the thernat conductivity of the alloy.

Thermal conductivity measurements were carried out on the thin composite films of embedded nanowires in a system characterized by its anisotropic thermal properties. Determination of the nanowire thermal conductivity is based on $2 D$ heat conduc- 

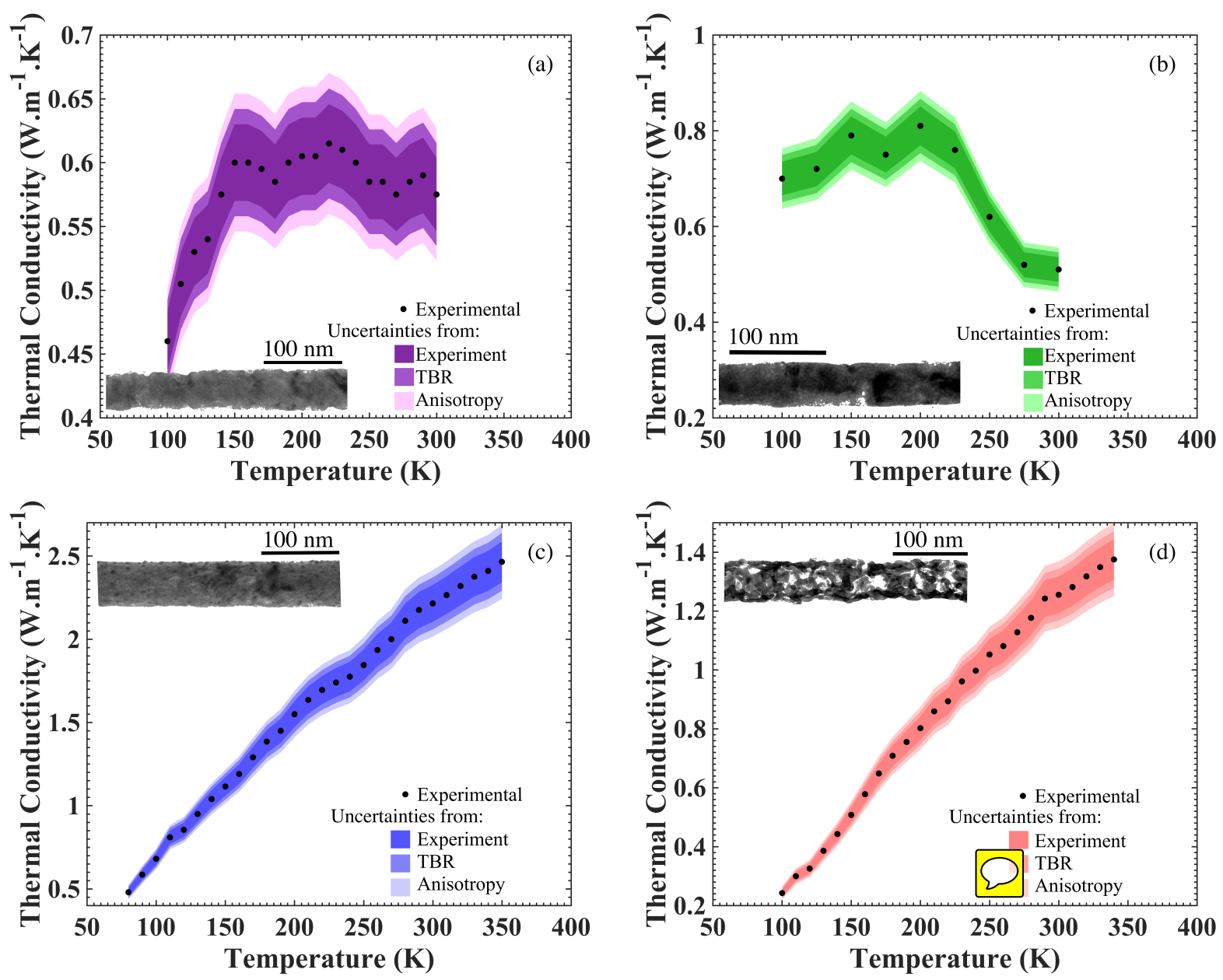

Figure 3: Temperature dependence of cross-plane thermal conductivity for (a) $\mathrm{Bi}_{0.6} \mathrm{Sb}_{1.85} \mathrm{Te}_{2.55}$ nanowires electro-deposited at $-0.3 \mathrm{~V}$, (b) $\mathrm{Bi}_{0.4} \mathrm{Sb}_{1.43} \mathrm{Te}_{3.17}$ nanowires deposited at $-0.15 \mathrm{~V}$, (c) $\mathrm{Bi}_{1.55} \mathrm{Te}_{2.9} \mathrm{Se}_{0.55}$ nanowires deposited at $0 \mathrm{~V}$ and, (d) $\mathrm{Bi}_{1.45} \mathrm{Te}_{2.85} \mathrm{Se}_{0.7}$ nanowire deposited at $-0.2 \mathrm{~V}$.

tion model for multilayer-on-finite substrate case (method for the determination of thermal conductivity is detailed in Supporting Information). The $3 \omega$ measurements were carried out from $100 \mathrm{~K}$ to $340 \mathrm{~K}$ in cryostat under high vacuum $\left(<10^{-5}\right.$ mbar) conditions. In Figure 3 , the temperature dependence of cross-plane thermal conductivity is plotted for the four different samples (two p-and two n-type which are of different stoichiometry). In the literature, the lattice component of bulk $\kappa$ for $\mathrm{Bi}_{2} \mathrm{Te}_{3}$ is $\approx 1.5 \mathrm{~W} \cdot \mathrm{m}^{-1} \cdot \mathrm{K}^{-1}$ in the plane perpendicular to c-axis $\left(\kappa_{a}\right.$, cleavage plane) and $\approx 0.7 \mathrm{~W} \cdot \mathrm{m}^{-1} \cdot \mathrm{K}^{-1}$ in the plane parallel to c-axis ${ }^{32}\left(\kappa_{c}\right)$ at $300 \mathrm{~K}$. The thermal conductivity depicted in the Figure 3 contains both the lattice and 
electronic contribution to the thermal transport. Hence, it is necessary to acquire the lattice component in order to compare any reduced thermal conductivity due to confinement effects in nanowires. The I-V neefficient measurements were perform $N W$ s deposited at $0 \mathrm{~V}$ and -0.2 $\mathrm{V}$ for $n$-type and -0.15 V and-0.3 $\mathrm{V}$ for p-type alloys. ${ }^{33}$

Table 1: Type of nanowire samples $70 \mathrm{~nm}$ in diameter, chemical compositions, electric and phonon lattice thermal conductivities along with the $Z T$ factor of merite at $300 \mathrm{~K}$.

\begin{tabular}{|c|c|c|c|c|c|c|}
\hline Sample & $\begin{array}{c}\text { Deposition } \\
\text { potential }(\mathrm{V})\end{array}$ & $\begin{array}{c}\text { Chemical } \\
\text { compositions }\end{array}$ & $\begin{array}{c}\mathrm{S}^{33} \\
(\mu \mathrm{V} / \mathrm{K})\end{array}$ & $\begin{array}{c}\rho_{e}^{33} \\
(\mathrm{~m} \Omega . \mathrm{cm})\end{array}$ & $\begin{array}{c}\kappa_{l} \\
\left(\mathrm{~W} \cdot \mathrm{m}^{-1} \cdot \mathrm{K}^{-1}\right)\end{array}$ & $Z T$ \\
\hline n-type & -0.2 & $\mathrm{Bi}_{1.45} \mathrm{Te}_{2.85} \mathrm{Se}_{0.7}$ & -39 & 0.956 & 0.6 & 0.07 \\
\hline $\mathrm{Bi}_{2} \mathrm{Te}_{3-\mathrm{y}} \mathrm{Se}_{\mathrm{y}}$ & 0 & $\mathrm{Bi}_{1.55} \mathrm{Te}_{2.9} \mathrm{Se}_{0.55}$ & -36 & 0.289 & 0.4 & 0.3 \\
\hline p-type & -0.15 & $\mathrm{Bi}_{0.4} \mathrm{Sb}_{1.43} \mathrm{Te}_{3.17}$ & +105 & 131 & 0.5 & 0.005 \\
\hline $\mathrm{Bi}_{2-\mathrm{x}} \mathrm{Sb}_{\mathrm{x}} \mathrm{Te}_{3}$ & -0.3 & $\mathrm{Bi}_{0.6} \mathrm{Sb}_{1.85} \mathrm{Te}_{2.55}$ & +108 & 527 & 0.5 & 0.001 \\
\hline
\end{tabular}

The electrical measurements were done with $N W s$ embedded in AAO as the latter being an insulator (electrical resistivity $10^{19} \Omega$ ), which is making sure that all the current passed through the NWs. The evolution of the resistivity was measured as a function of temperature using the Physical Property Measurement System (PPMS). The embedded nanowire film was annealed at $523 \mathrm{~K}$ under $\mathrm{Ar} / \mathrm{H}_{2}$ for four hours to improve the crystalline structure. Multiple nanowires were connected through the contact pad made of aluminium, where the number of nanowires were estimated using the porosity and pore-filling ratios. The resistivity of the single nanowire was calculated by assuming that each of the nanowires has the same resistance and connected in parallel. However, errors may arise in the estimation of the resistivity of single nanowire from the unaccounted broken nanowires while deducing it from the measurement on the set of nanowires. The correlation between morphology and electrical conductivity is relatively complex. However, it is easy to understand that the electrical as the grain size increases, because the mobility is enhanced by reducing the carrier scattering on the grain boundaries as the grains grow. ${ }^{9}$ In addition, bismuth telluride is known to have anisotropic electrical conductivity which depends on the alloy composition. Any deviation in crystallite orientation from the expected crystal and 
any improper doping (anti-site) could strongly influence the material properties. ${ }^{34}$ Especially in the case of polycrystalline p-type NWs, the large number of grain boundaries and crystalline structure defects should play a major role in the increase of electrical resistivity. ${ }^{35,36}$ The Seebeck coefficient was also experimentally derived for the whole array of NWs by using overgrown nanowires from the templates as good thermal and electrical contact. ${ }^{37}$ The low Seebeck value, in comparison with bulk and films, ${ }^{9}$ is due to a high carrier density induced by the strong deficit in bismuth which leads to the creation of anti-site Te electron donor defects. ${ }^{38}$ A similar effect is observed for p-type NWs. Moderate Seebeck values are obtained because of an excess in charge carrier concentration induced by the large density of anti-site defects currently obtained in BiSbTe systems. For NWs, it is very difficult to correlate the Seebeck coefficient value to the composition, especially for polycrystalline nanowires, since this would require the measurement of carrier concentration, which is not an easy task.

The Wiedemann-Franz law $\left(\kappa_{e}=\sigma L_{0} T\right)$ was used to calculate the electronic contribution to the thermal conductivity from the electrical conductivity and hence deduce the phononic (lattice) component of thermal conductivity. Usually, Lorentz number, $L_{0}$, is considered as a universal constant of $2.44 \times 10^{-8}{\mathrm{~W} \Omega \mathrm{K}^{-2}}^{-1 e r i v e d ~ f o r ~ t h e ~ d e g e n e r a t e ~ l i m i t, ~ h o w e v e r, ~ s i g-~}$ nificant deviations occur for non-degenerate semiconductors which is often related to the increased thermopower $S .{ }^{39-42}$ Important information to take note here is that the $L_{0}$ depends on the band structure, position of the Fermi level and temperature - in the case of semiconductors this leads to carrier concentration. In the past, analytical solution of $L_{0}$ based on the single parabolic band (SPB) model obtained by solving the Boltzmann transport equations (BTE) have been often employed. One such approximate solution was provided by Flage-Larsen et al. ${ }^{43}$ based on the SPB model as a function of carrier concentration $n$ and $m * T^{3 / 2}$ ( $\left.m *\right)$ is the effective mass) along with multiple parameter sets of distinct mechanisms for scattering. Recently, it has been seen that the correlation between $L_{0}$ and the measured $S$ of the material can be exploited to estimate the $\kappa_{L}$ with better accuracy for different thermoelectric materials. ${ }^{39-42}$ A first order correction based on Seebeck coefficient can be applied 
to the degenerate model assuming a single parabolic band/acoustic phonon scattering. ${ }^{44}$ The equation, $L_{0}=1.5+\exp \left(\frac{-|S|}{116}\right)$ (where $L_{0}$ is in $10^{-8} \mathrm{~W}_{\Omega} \mathrm{K}^{-2}$ and $S$ is in $\mu \mathrm{V} / \mathrm{K}$ ), is considered to be a satisfactory assumption. 44

The extracted cross-plane phononic thermal conductivity, as obtained from the data treatment explained above, is compared for all four samples in Figure 4 a to the thermal conductivity of a mix of c-axis and a-axis of $\mathrm{Bi}_{2} \mathrm{Te}_{3}$ computed by solving the Boltzmann Transport Equation in the frame of the relaxation time approximation. The first thing that we learn is a reduction of thermal conductivity to $0.6 \mathrm{~W} \cdot \mathrm{m}^{-1} \cdot \mathrm{K}^{-1}$ as compared to $1.5 \mathrm{~W} \cdot \mathrm{m}^{-1} \cdot \mathrm{K}^{-1}$ and $0.7 \mathrm{~W} . \mathrm{m}^{-1} \cdot \mathrm{K}^{-1}$ for two major axis of the bulk for $\mathrm{T}=200 \mathrm{~K}$. However, since the averaged bulk phonon mean free path of bismuth telluride is only $3 \mathrm{~nm},{ }^{45}$ this reduction of thermal conductivity cannot be attributed only to the small diameter of nanowires (70 $\mathrm{nm}$ ).

The numerical simulations, as shown in Figure 4 a were done using the Monte Carlo technique to compute the thermal conductivity for $B i_{2} \mathrm{Te}_{3}$ in a nanowire of $70 \mathrm{~nm}$ in diameter and $1 \mu \mathrm{m}$ of length. The latter thermal conductivities in nanowire and bulk were obtained along a and c-axis, and were averaged in order to replicate the random distribution of the two main axis. This shows that part of the reduction in thermal transport comes from the reduction of the nanowire diameter. However, as the temperature is decreased, a significant departure from the simulation is observed which is associated to enhanced phonon-boundary scattering on the rough nanowire surfaces. Another explanation of the low temperature discrepancies might arise from the alloying disorder or grain boundary scattering which is not taken into account in the Monte Carlo modeling. When temperature is decrease these scattering mechanisms shall hinder phonon with larger mean free path, while Normal and Umklapp mechanisms dominate at higher temperatures. Details about the Monte Carlo simulations ${ }^{6} 6$ are provided in the Supporting Information.

When comparing the phonon thermal conductivity of the four different bismuth telluride nanowires under the criterion of surface roughness, a pronounced effect exists at low temperatures, with a significant decrease of $\kappa(T)$ especially in $\mathrm{Bi}_{1.45} \mathrm{Te}_{2.85} \mathrm{Se}_{0.7}$. Both $\sigma$ and $l_{\text {corr }}$ 


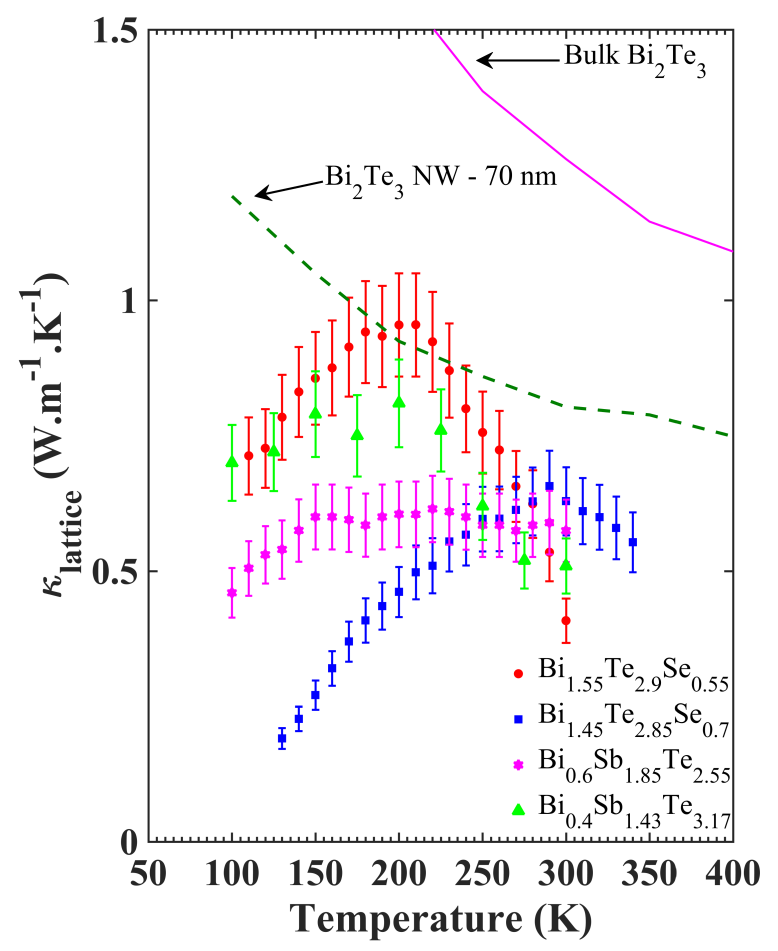

(a)

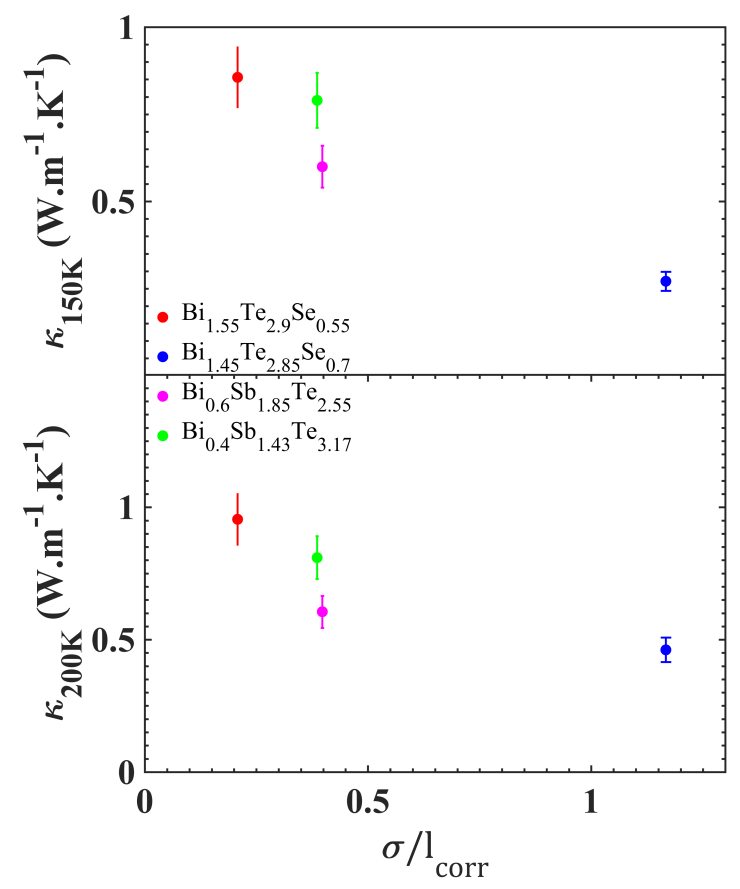

(b)

Figure 4: (a) Lattice thermal conductivity, as calculated from the experimental values of total thermal conductivity. (b) Variation of the thermal conductivity at two different temperatures $150 \mathrm{~K}$ and $200 \mathrm{~K}$ as a function of the ratio between the roughness and the correlation length. 
determines the surface roughness of the nanowires, however, the thermal conductivity reduction is strongly related to the factor $\sigma / l_{\text {corr }}$ as shown in Figure $4 b$. The enhanced reduction from roughness could possible arise from the backscattering of phonons or due to trapping of phonons between the roughness features by multiple boundary scatterings. Lim et al. also proved for silicon nanowires that the factor of $\sigma / l_{\text {corr }}$ hold more importance in determining the reduction in thermal conductivity rather than the $\sigma$ or $l_{\text {corr }}$ alone. ${ }^{29}$ It is striking to note that in our experiment even if the mean free path of phonon is much smaller than the nanowire diameter, the effect of the factor $\sigma / l_{\text {corr }}$ on the decrease of thermal conductivity still hold.

In conclusion, temperature dependence of thermal conductivity has been established using $3 \omega$ method for embedded nanowires of $\mathrm{Bi}_{2} \mathrm{Te}_{3-y} S e_{y}$ and $\mathrm{Bi}_{2-x} S b_{x} \mathrm{Te}_{3}$ nanowire forest. Sizable decrease in thermal conductivity is observed in nanowires of diameter $70 \mathrm{~nm}$ at room temperature as compared to bulk, but, enhanced reduction in thermal conductivity is shown for lower temperature. The reduction in the thermal conductivity as compared to the bulk is strongly linked to the reduction of the diameter but also to the surface roughness dimensionless factor of $\sigma / l_{\text {corr }}$. A correlation is shown between $\sigma / l_{\text {corr }}$ and the decrease of thermal conductivity, a signature of significant phonon scattering on surface roughness. This shows that low thermal conductivities can be obtained for large number of nanowires embedded in alumina matrix. Further efforts in improving the electrical conductivity along with increased roughness with this $3 D$ cross-plane architecture of nanowires could lead to new concept of macroscopic thermoelectric devices based on nanowires.

\section{Acknowledgement}

The authors thank the technical support and discussions provided by the Nanofab, Pôle Capteur, Pôle électronique, Póle cryogenic facilities in Institut Néel and Plateforme Technologique Amont (PTA) in CEA. The authors are grateful to the funding and support from 
the ANR project MESOPHON, the Laboratoire dexcellence LANEF in Grenoble (No. ANR10-LABX-51-01), and from the ANRT. The authors would also like to thank ST Microelectronics for collaboration on part of this work. ECA, JJF and CMST acknowledge support from the Severo Ochoa Program (MINECO, Grant SEV-2013-0295) and funding from the CERCA Programme/Generalitat de Catalunya. Funding from the Spanish Ministry MINECO/FEDER: FIS2015-70862-P PHENTOM is also acknowledged.

\section{References}

(1) Dresselhaus, M.; Lin, Y.-M.; Koga, T.; Cronin, S.; Rabin, O.; Black, M.; Dresselhaus, G.; Tritt, T. Semiconductors and Semimetals: Recent Trends in Thermoelectric Materials Research III. Academic, San Diego, CA 2001,

(2) Tritt, T. M. Thermoelectrics run hot and cold. Science 1996, 272, 1276-1277.

(3) Tritt, T. M. Holey and unholey semiconductors. Science 1999, 283, 804-805.

(4) Selvan, K. V.; Ali, M. S. M. Micro-scale energy harvesting devices: Review of methodological performances in the last decade. Renewable \& Sustainable Energy Reviews 2016, 54, 1035-104\%.

(5) Davila, D.; Tarancon, A.; Calaza, C.; Salleras, M.; Fernandez-Regulez, M.; San Paulo, A.; Fonseca, L. Monolithically integrated thermoelectric energy harvester based on silicon nanowire arrays for powering micro/nanodevices. Nano Energy 2012, 1, 812-819.

(6) Perez-Marin, A.; Lopeandia, A.; Abad, L.; Ferrando-Villaba, P.; Garcia, G.; Lopez, A. M.; Munoz-Pascual, F. X.; Rodriguez-Viejo, J. Micropower thermoelectric generator from thin Si membranes. Nano Energy 2014, 4, 73-80. 
(7) Tainoff, D.; Proudhom, A.; Tur, C.; Crozes, T.; Dufresnes, S.; Dumont, S.; Bourgault, D.; Bourgeois, O. Network of thermoelectric nanogenerators for low power energy harvesting. Nano Energy 2019, 57, 804.

(8) Dresselhaus, M.; Dresselhaus, G.; Sun, X.; Zhang, Z.; Cronin, S.; Koga, T.; Ying, J.; Chen, G. The promise of low-dimensional thermoelectric materials. Microscale Thermophysical Engineering 1999, 3, 89-100.

(9) Nolas, G. S.; Sharp, J.; Goldsmid, H. J. Thermoelectrics; Springer, 2001; pp 111-176.

(10) Bourgeois, O.; Tainoff, D.; Tavakoli, A.; Liu, Y.; Blanc, C.; Boukhari, M.; Barski, A.; Hadji, E. Reduction of phonon mean free path: From low-temperature physics to room temperature applications in thermoelectricity. COMPTES RENDUS PHYSIQUE 2016, 17, 1154-1160.

(11) Rowe, D. M. CRC Handbook of Thermoelectrics; CRC press, 1995; pp 31-37.

(12) Borca-Tasciuc, D.-A.; Chen, G.; Prieto, A.; Martín-González, M. S.; Stacy, A.; Sands, T.; Ryan, M.; Fleurial, J. Thermal properties of electrodeposited bismuth telluride nanowires embedded in amorphous alumina. Appl. Phys. Lett. 2004, 85, 6001-6003.

(13) Zhou, J.; Jin, C.; Seol, J. H.; Li, X.; Shi, L. Thermoelectric properties of individual electrodeposited bismuth telluride nanowires. Appl. Phys. Lett. 2005, 87, 133109.

(14) Rojo, M. M.; Abad, B.; Manzano, C.; Torres, P.; Cartoixà, X.; Alvarez, F.; Gonzalez, M. M. Thermal conductivity of Bi 2 Te 3 nanowires: How size affects phonon scattering. Nanoscale 2017, 9, 6741-6747.

(15) Lin, Y.-M.; Sun, X.; Dresselhaus, M. Theoretical investigation of thermoelectric transport properties of cylindrical Bi nanowires. Phys. Rev. B 2000, 62, 4610. 
(16) Slack, G. A. New materials and performance limits for thermoelectric cooling. CRC handbook of thermoelectrics 1995, 407-440.

(17) Swinkels, M.; Zardo, I. Nanowires for heat conversion. J. Phys. D: Appl. Phys. 2018, $51,353001$.

(18) Wang, W.; Jia, F.; Huang, Q.; Zhang, J. A new type of low power thermoelectric micro-generator fabricated by nanowire array thermoelectric material. Microelectronic Engineering 2005, 77, 223-229.

(19) Curtin, B. M.; Fang, E. W.; Bowers, J. E. Highly ordered vertical silicon nanowire array composite thin films for thermoelectric devices. Journal of Electronic Materials 2012, 41, 887-894.

(20) Yuan, Z.; Ziouche, K.; Bougrioua, Z.; Lejeune, P.; Lasri, T.; Leclercq, D. A planar micro thermoelectric generator with high thermal resistance. Sensors and Actuators A: Physical 2015, 221, 67-76.

(21) Khedim, M. B.; Cagnon, L.; Serradeil, V.; Fournier, T.; Bourgault, D. Thermoelectric nanowires based on bismuth telluride. Materials Today: Proceedings 2015, 2, 602-609.

(22) Muñoz Rojo, M.; Grauby, S.; Rampnoux, J.-M.; Caballero-Calero, O.; MartínGonzález, M.; Dilhaire, S. Fabrication of Bi2Te3 nanowire arrays and thermal conductivity measurement by 3w-scanning thermal microscopy. J. Appl. Phys. 2013, 113, 054308.

(23) Prieto, A. L.; Sander, M. S.; Martín-González, M. S.; Gronsky, R.; Sands, T.; Stacy, A. M. Electrodeposition of ordered Bi2Te3 nanowire arrays. Journal of the American Chemical Society 2001, 123, 7160-7161.

(24) Singhal, D.; Paterson, J.; Tainoff, D.; Richard, J.; Ben-Khedim, M.; Gentile, P.; Cagnon, L.; Bourgault, D.; Buttard, D.; Bourgeois, O. Measurement of anisotropic 
thermal conductivity of a dense forest of nanowires using the $3 \omega$ method. Review of Scientific Instruments 2018, 89, 084902.

(25) Buff, H. Ueber das electrische Verhalten des Aluminiums. Liebigs Ann. Chem. 1857, 102, 265-284.

(26) Masuda, H.; Satoh, M. Fabrication of gold nanodot array using anodic porous alumina as an evaporation mask. Jpn. J. Appl. Phys. 1996, 35, L126.

(27) Lee, W.; Park, S.-J. Porous anodic aluminum oxide: anodization and templated synthesis of functional nanostructures. Chemical Reviews 2014, 114, 7487-7556.

(28) Menke, E.; Brown, M.; Li, Q.; Hemminger, J.; Penner, R. Bismuth telluride (Bi2Te3) nanowires: Synthesis by cyclic electrodeposition/stripping, thinning by electrooxidation, and electrical power generation. Langmuir 2006, 22, 10564-10574.

(29) Lim, J.; Hippalgaonkar, K.; Andrews, S. C.; Majumdar, A.; Yang, P. Quantifying surface roughness effects on phonon transport in silicon nanowires. Nano Letters 2012, 12, $2475-2482$.

(30) Goodnick, S.; Ferry, D.; Wilmsen, C.; Liliental, Z.; Fathy, D.; Krivanek, O. Surface roughness at the Si (100)-SiO 2 interface. Phys. Rev. B 1985, 32, 8171.

(31) Yamakawa, S.; Ueno, H.; Taniguchi, K.; Hamaguchi, C.; Miyatsuji, K.; Masaki, K.; Ravaioli, U. Study of interface roughness dependence of electron mobility in Si inversion layers using the Monte Carlo method. J. Appl. Phys. 1996, 79, 911-916.

(32) Goldsmid, H. The thermal conductivity of bismuth telluride. Proceedings of the Physical Society. Section B 1956, 69, 203.

(33) Khedim, M. B.; Cagnon, L.; Garagnon, C.; Serradeil, V.; Bourgault, D. Direct electrical transport measurement on a single thermoelectric nanowire embedded in an alumina template. Physical Chemistry Chemical Physics 2016, 18, 12332-1233\%. 
(34) Ivanova, L.; Granatkina, Y. V.; Polikarpova, N.; Smirnova, E. Selenium-doped Sb2Te3Bi2Te3 Crystals. Inorganic Materials 1997, 33, 558-561.

(35) Miller, G.; Li, C.-Y. Evidence for the existence of antistructure defects in bismuth telluride by density measurements. Journal of Physics and Chemistry of Solids 1965, $26,173-177$.

(36) Kavei, G.; Karami, M. Fabrication and characterization of the p-type (Bi 2 Te 3) $x$ (Sb 2 Te 3) 1-x thermoelectric crystals prepared via zone melting. Bulletin of Materials Science 2006, 29.

(37) Klammer, J.; Bachmann, J.; Töllner, W.; Bourgault, D.; Cagnon, L.; Gösele, U.; Nielsch, K. Electrochemical route to thermoelectric nanowires via organic electrolytes. Physica Status Solidi (b) 2010, 247, 1384-1392.

(38) Liao, C.-N.; She, T.-H. Preparation of bismuth telluride thin films through interfacial reaction. Thin Solid Films 2007, 515, 8059-8064.

(39) May, A. F.; Fleurial, J.-P.; Snyder, G. J. Thermoelectric performance of lanthanum telluride produced via mechanical alloying. Phys. Rev. B 2008, 78, 125205.

(40) Fu, C.; Liu, Y.; Xie, H.; Liu, X.; Zhao, X.; Jeffrey Snyder, G.; Xie, J.; Zhu, T. Electron and phonon transport in Co-doped FeV0.6Nb0.4Sb half-Heusler thermoelectric materials. J. Appl. Phys. 2013, 114, 134905.

(41) Zevalkink, A.; Zeier, W. G.; Pomrehn, G.; Schechtel, E.; Tremel, W.; Snyder, G. J. Thermoelectric properties of Sr3GaSb3-a chain-forming Zintl compound. Energy \& Environmental Science 2012, 5, 9121-9128.

(42) Pei, Y.; LaLonde, A.; Iwanaga, S.; Snyder, G. J. High thermoelectric figure of merit in heavy hole dominated PbTe. Energy \& Environmental Science 2011, 4, 2085-2089. 
(43) Flage-Larsen, E.; Prytz, Ø. The Lorenz function: Its properties at optimum thermoelectric figure-of-merit. Appl. Phys. Lett. 2011, 99, 202108.

(44) Kim, H.-S.; Gibbs, Z. M.; Tang, Y.; Wang, H.; Snyder, G. J. Characterization of Lorenz number with Seebeck coefficient measurement. APL Materials 2015, 3, 041506.

(45) Mavrokefalos, A.; Moore, A. L.; Pettes, M. T.; Shi, L.; Wang, W.; Li, X. Thermoelectric and structural characterizations of individual electrodeposited bismuth telluride nanowires. J. Appl. Phys. 2009, 105, 104318.

(46) Lacroix, D.; Joulain, K.; Terris, D.; Lemonnier, D. Monte Carlo simulation of phonon confinement in silicon nanostructures: Application to the determination of the thermal conductivity of silicon nanowires. Appl. Phys. Lett. 2006, 89, 103104. 